\title{
The response of denervated muscle to long-term stimulation (1985, revisited here in 2014)
}

\author{
Terje Lomo
}

Institute of Basic Medical Sciences, University of Oslo, Norway

\begin{abstract}
In 1985, at a meeting in Abano, I presented results showing that direct stimulation of skeletal muscles with appropriate stimulus patterns prevents the effects of denervation on nonjunctional properties of muscle fibers. Hence, it appeared unnecessary to postulate that unknown nerve-derived trophic factors control such properties, as posited by the (anterograde) neurotrophic hypothesis. Here I discuss this conclusion in the light of what we know today, particularly with respect to the many lines of evidence that were then taken to support the trophic hypothesis, but which today have alternative interpretations consistent with control by evoked impulse activity. Despite much effort, no one has yet identified any nerve-derived factor consistent with the neurotrophic hypothesis. Reports favoring the existence of neurotrophic factors were numerous before 2000. Now they have essentially disappeared from the literature, including original research papers, textbooks and handbooks, suggesting that the hypothesis is no longer arguable. Thus, the results that I presented in our paper in 1985 seem to have held up rather well.
\end{abstract}

Key Words: skeletal muscle, neurotrophic hypothesis, denervation, ACh supersensitivity, fast/slow contractile characteristics, electrical stimulation

Eur J Trans Myol - Basic Appl Myol 2014; 24 (1): 13-19

\begin{abstract}
At a meeting in Abano in 1985 I presented a paper with this title. ${ }^{1}$ Here, I discuss that paper in the light of previous and present knowledge and ask how its conclusions stand today. In the 1985 paper I concluded that nerve evoked muscle impulse activity is essential for the control of extrajunctional membrane and contractile properties of skeletal muscle fibres and, consequently, that loss of such activity explains why denervated muscle fibres become sensitive to acetylcholine $(\mathrm{ACh})$ along their entire length (ACh supersensitivity). I further argued that crossreinnervated fast muscles become slow because they now receive slow impulse patterns, while slow muscles become fast because they receive fast impulse patterns.
\end{abstract}

\section{The Neurotrophic Hypothesis before 1972}

My view that loss of nerve evoked muscle impulse activity explains the effects of denervation on nonjunctional muscle properties was not generally accepted in 1985. Before 1972 many researchers addressing the problem thought that trophic factors released from motor nerve terminals in muscle were responsible (see below). After 1972, many concluded that both impulse activity and trophic factors were important.

Perhaps the first to distinguish between evoked impulse activity and trophic factors in the regulation of muscle properties was Sarah Tower in $1937 .^{2}$ Sparing only the ventral roots, she isolated the lumbo-sacral spinal cord in kittens to "silence" the muscle and compare inactive innervated muscles with denervated muscles. Referring to others ${ }^{3,4}$ and her own observations she wrote: "Therefore, since atrophy is apparently inevitable after denervation, whether the muscle be artificially activated or not, both activity and some other as yet unrecognized trophic agent must operate between nerve and muscle beyond the motor end-plate".

More evidence for trophic agents emerged around 1960 when Ricardo Miledi reported that ACh supersensitivity appeared around denervated endplates in multiply innervated frog muscle fibres kept active by intact innervation elsewhere on the same muscle fibres and, further, that the ACh supersensitivity started to disappear before evoked impulse activity was observed during nerve regeneration, findings which led him to conclude "that the neural factor which controls 


\section{The response of denervated muscle to long-term electrical stimulation}

Eur J Trans Myol - Basic Appl Myol 2014; 24 (1): 13-19

the number and spread of acetylcholine receptors in the muscle fibres is independent of nerve impulses" ${ }^{\text {. 5,6 }}$ At about the same time, Stephen Thesleff and coworkers reported that little or no ACh supersensitivity appeared in muscle fibres inactivated by spinal cord isolation. ${ }^{7}$ But since the supersensitivity did appear after neuromuscular transmission block by botulinum toxin, ${ }^{8}$ they proposed that spontaneously released ACh could be the trophic agent. Before that, Luco and Eyzaguirre reported that when nerves were cut at increasingly longer distances from the muscle, the appearance of ACh supersensitivity and fibrillations was progressively delayed, ${ }^{9}$ presumably because nerve terminal degeneration, ${ }^{10}$ and hence interruption of nerve-released trophic factors, is delayed when the stump is long. Such findings then led John Eccles to write in The Physiology of Synapses (1964): "it can be stated that the evidence for a trophic influence from nerve onto muscle is conclusive, but it is still uncertain whether it is entirely affected by unique trophic influences or whether in part by the spontaneous emission of quanta of ACh". ${ }^{11}$

In their classic cross-reinnervation experiments in 1960, Buller, Eccles and Eccles concluded: "the neural influence on muscle speed is not exerted by nerve impulses as such. It is postulated that a substance passes down the axons of slow motoneurons, crosses the neuromuscular junctions and traverses the muscle fibres, transforming them into slow contraction units and maintaining them so. Possibly there is also a substance from fast motoneurons that acts via a comparable pathway to accelerate muscle contraction". ${ }^{12}$ In a later paper Eccles and coworkers mention the possibility of "a specific influence of frequency of motoneuron discharge" but then only indirectly as a reaction to "the vibratory stress of activation at a frequency of about 10/sec, an explanation suggested by Professor A.F. Huxley". ${ }^{13}$ That different patterns of impulse activity by themselves might alter the speed of contraction of muscles was not addressed.

\section{Direct stimulation of denervated muscle}

In 1969-1971, Jean Rosenthal and I, working as postdocs in The Department of Biophysics at University College London headed by Bernard Katz and Ricardo Miledi, obtained results that appeared to turn the idea of essential trophic factors upside down. ${ }^{14}$ Stimulating denervated rat soleus (SOL) and extensor digitorum longus (EDL) muscles directly through chronically implanted electrodes, we showed that the stimulation either prevented ACh supersensitivity from developing or caused it to disappear if the stimulation started when the fibres were already supersensitive. Later in Oslo, my colleagues and I showed that such stimulation also restored other membrane properties to normal. ${ }^{15,16}$ Furthermore, when we stimulated the slow SOL with patterns that resembled the firing patterns of fast motor neurons and the fast EDL with patterns that resembled the firing patterns of slow motor neurons, we essentially reproduced the effects of crossreinnervation. ${ }^{17-20}$ In these experiments no motor nerve terminals were present in the muscles and there was no way that trophic factors released from them could explain the effects. Instead, it seemed to me, the different patterns of evoked muscle activity had to be solely responsible.

\section{The neurotrophic hypothesis after 1972}

Many researchers either did not accept our results or they thought of trophic factors as essential but acting in conjunction with evoked impulse activity. Here are some examples of the arguments raised against our work and the idea that loss of evoked muscle activity alone is responsible for the effects of denervation on muscle.

In 1976, Deshpande, Albuquerque, and Guth concluded that "both the prejunctional nerve membrane and the postjunctional muscle membrane are regulated by a neurohumoral factor". In addition, they wrote: "Furthermore, the experiments using prolonged electrical stimulation (Lфmo and Westgaard, 1975; ${ }^{15}$ Westgaard, 1975 ${ }^{16}$ ) can be criticised because they were performed on chronically denervated muscles. The denervated muscle fiber is a cell that has been released from many of its physiological regulatory controls; given our present state of knowledge one cannot use such pathological tissue to make inferences about the role of muscle activity on physiologically normal muscle fibers; this experimental approach appears to us to be inappropriate for the study of trophic nerve function". 21

This critique seemed strange. It is common research practice to remove some unidentified factor and then add a specific, identified factor to see whether it can replace the unidentified one and restore normal function, which is what direct stimulation does.

In 1976, Ernst Gutmann discussed "Increasing evidence for the existence of neurotrophic (nonimpulse) mechanisms, especially in nerve-muscle cell relations". ${ }^{22}$ In 1981 Sreter and co-workers concluded: "There is no evidence that the fast activity pattern plays a significant role in determining fast-twitch muscle properties" ,23 suggesting that our stimulations had damaged the muscles and that new fiber formation explained our results. In 1991, Witzeman et al. write that "levels of the $\gamma$-subunit in the entire fiber [a sign of ACh supersensitivity] are reduced by a negative neural factor and possibly also by nerve-induced electrical muscle activity". ${ }^{24}$ In 1994, in the Handbook Myology (1994), Alan Grinnell writes: "Because the directstimulation experiments are so convincing, it is tempting to conclude that muscle activity is the only factor responsible for many of the muscle properties changed by denervation or that it is essential to their 
regulation. However, there is compelling evidence that activity-based effects are superimposed on at least two other mechanism of trophic regulation". ${ }^{25}$ And in 2001, the textbook "From Neuron to Brain" cites Witzemann et al. (1991) as evidence for additional trophic factors. ${ }^{26}$

\section{Trophic factors versus products of nerve degeneration}

In his review in Myology (1994), Grinnell discussed the evidence for trophic regulation under the headings: "Stump length effects," "Nerve breakdown products," "Nerve conduction block," "Block of axonal transport," and "Pharmacological block of synaptic transmission." Why did this evidence appear compelling? An important reason was the observation that blockage of impulse conduction in an otherwise intact nerve initially causes less ACh supersensitivity (and lower levels of other denervation-like changes) than denervation.

Since both interventions block evoked muscle activity at the same time, inactivity alone could not be responsible for all the effects of denervation. According to the pro-trophic interpretation, continued release of trophic factors from intact but "silent" motor nerve terminals was responsible. According to the proactivity interpretation, nerve terminal degeneration and accompanying reactions in the muscle were responsible, as such reactions would boost the response of muscle fibres to inactivity.

Alberto Cangiano and I have called such reactions "Products of nerve degeneration". The identity of such products is unknown, but would include molecules (products) that degenerating axons and nerve terminals or denervated muscle fibres generate or immune and tissue cells activated by such molecules produce. Support for this idea comes from the observation that denervation elicits proliferation and accumulation of tissue cells, including macrophages, in affected muscles both in the frog and rat ${ }^{27-29}$. The response is most pronounced in the junctional region but affects the entire length of the muscle fibres. It is also transient with maximal signs of cell proliferation after 4 days, which then subside to control values. Importantly, no such tissue reactions are seen after nerve conduction blocks. ${ }^{28}$ Thus, Murray and Robbins (1982) conclude: "cell proliferation after denervation is not a response to simple disuse, but rather to a nerve- or musclerelated mitogen".

The responses of muscle to denervation have similarities to inflammation, and acute inflammation induces transient $\mathrm{ACh}$ supersensitivity in innervated muscle fibres, as observed in muscles subjected to surgical traumas or presence of foreign bodies or degenerating nerves on their surface. ${ }^{15,30-32}$ However, not only inflammation, but any of the products of nerve degeneration mentioned above could induce ACh supersensitivity and other muscle fiber changes. For the pro-activity interpretation to be correct, the greater muscle response to cutting axons compared to just blocking their impulse conduction should be transient, like the mitogenic effects of denervation on the muscle. In a series of papers, Alberto Cangiano's lab has produced what I take as compelling evidence that this is the case. ${ }^{33-36}$ Thus, denervation markedly speeds up and potentiates muscle fiber responses to inactivity by factors that act only during the first 1-2 weeks of denervation. After that, as convincingly shown by Cangiano, the denervation-like effects of nerve impulse block and denervation are equal ${ }^{35,37}$ Moreover, when a nerve with impulse conduction completely blocked by TTX reinnervates a denervated muscle, no reduction in ACh supersensitivity (or TTX resistance of muscle action potentials) is observed as the neurotrophic hypothesis would predict. ${ }^{35}$

Such results offer a very different interpretation of the types of experiments listed by Grinnell as compelling evidence for trophic factors. Thus, "Stump length effects" occur because nerve degeneration occurs earlier the shorter the distal nerve stump is, ${ }^{10,31}$ not because trophic factors disappear earlier. "Nerve conduction block" is initially less effective than denervation because of the absence of inflammatory or inflammatory-like responses, ${ }^{28}$ ] not because "silent" nerve terminals continue to release trophic factors. Transient "products of nerve degeneration" also explains the local supersensitivity observed by Miledi around denervated endplates in multiply innervated frog muscle fibres. "Block of axonal transport" relates to the moderate $\mathrm{ACh}$ supersensitivity and other denervation-like changes observed after applying colchicine to the sciatic nerve, effects that were attributed to blockage of axonal transport by colchicine, including trophic factors. However, as Cangiano and I showed independently, colchicine acts systemically, affecting innervated muscles in different parts of the body equally regardless of where colchicine is injected. ${ }^{38,39}$ In addition, I showed that colchicine affects innervated and denervated stimulated muscle fibres equally, which shows that it probably affects muscle directly and certainly by mechanisms independent of the nerve. ${ }^{39}$ "Pharmacological block of synaptic transmission" refers in part to "Witzeman et al. (1991), who used tetrodotoxin (TTX) to block nerve impulse conduction and alpha-bungarotoxin and injection of botulinum toxin into the muscle to block neuromuscular transmission, and who concluded in favor of "a negative [trophic] neural factor". ${ }^{4}$ However, the effects of TTX that they observed were surprisingly small and those of botulinum toxin surprisingly large. Therefore, the TTX block might have been incomplete, and the injection of botulinum toxin might have elicited an inflammation that boosted the effect of inactivity per se. In my view, these are real possibilities because complete blocks of the rat sciatic nerve by TTX are difficult to obtain and require 
special precautions, ${ }^{35,37}$ and because botulinum toxin is a foreign protein likely to cause inflammation when injected into a muscle.

\section{Trophic interactions as understood today}

The neurotrophic hypothesis discussed here postulated nerve-derived factors acting anterogradely onto entire muscle fibres. The factors were trophic ("nutritional") in the sense that in their absence muscle fibres would atrophy and eventually disappear. Today, lacking firm support and presence in recent literature, the hypothesis appears discarded. But motor nerve terminals do release other substances with other essential functions, for example, agrin, which sets up the postsynaptic apparatus, causes its differentiation and separation from the extrajunctional membrane, and elicits the expression of postsynaptic factors that signal back to regulate the differentiation of presynaptic terminals. ${ }^{40}$ But because agrin affects only the junction, it is not a trophic factor in the original sense. In the peripheral nervous system, the demonstration of nerve growth factor (NGF) in target tissues that act back to promote the survival and maintenance of sensory- and sympathetic neurons has led to a generally accepted neurotrophic theory based on retrograde signaling. ${ }^{41,42}$ Motor neurons also depend on their target (muscle) for their survival, ${ }^{43}$ but unique trophic factors have not been demonstrated, evidently because different subpopulations of motor neurons respond differently to many candidate factors. ${ }^{44}$ In the brain, both presynaptic nerve terminals and postsynaptic dendrites appear to secrete brain derived neurotrophic factor (BDNF) in activity dependent manners with effects that may be local in its regulation of synaptic efficiency at particular dendritic sites, or more general in its regulation of protein synthesis, neurite and dendritic growth. ${ }^{45,46}$ Secreted from pre-and postsynaptic sites, BDNF may therefore act both anterogradely and retrogradely.

\section{Stimulating innervated versus denervated muscle}

It is also important to distinguish between effects of stimulating denervated muscles directly and stimulating innervated muscles indirectly via intact motor axons. Much work has shown that indirect stimulation can markedly affect the contractile properties of muscle. ${ }^{47-49}$ However, this approach does not resolve the issue of trophic factor versus evoked impulse activity because with intact motor neurons it is impossible to rule out an effect of stimulation on the production, transport, composition, or release of putative trophic factors. Moreover, since the selfgenerated impulse activity of the intact motor neurons is not recorded, the precise pattern of impulses imposed on the muscle is unknown.

\section{Advantages of stimulating denervated muscles}

For many years my lab was the only one that performed chronic stimulation of denervated muscle. Using this approach, we not only could exclude nervederived trophic influences but also obtain complete control of the stimulus patterns imposed on the muscle. I believe we were the first to study systematically the role of different impulse patterns in regulating the properties of postsynaptic cells. ${ }^{15,18-20}$ By varying the frequency and number of stimuli as well as the duration and timing of individual stimulus trains we described how different stimulus patterns affected not only the rate of disappearance of ACh supersensitivity, but also the speed of contraction of muscles and their expression patterns of myosin heavy chain and fiber types, ${ }^{19}$ as well as many other muscle properties. Because SOL and EDL responded in strikingly different ways to identical stimulus patterns, we introduced the concept of "adaptive range", which posits that patterns of impulse activity regulate the contractile properties of muscle fibres in a graded manner within different intrinsic limits in different types of muscle fibres. ${ }^{18}$ By mixing fast and slow stimulus patterns, we obtained intermediate contractile speeds, consistent with graded regulation and interactions between intracellular pathways towards slow or fast muscles. We explained why spontaneous activity (fibrillations) in denervated muscle fibres had little effect on their extrajunctional ACh sensitivity by delivering a given number of stimulus trains over a relatively short period of time (6 hours) every 24 hours or spread out over 24 hours. Like fibrillations, which consist of relatively brief periods of activity interrupted by much longer periods of inactivity, the stimulus trains had only a small effect when delivered over 6 hours. When spread over 24 hours the effect was very strong. ${ }^{15}$ Thus, to avoid denervation-like reactions any single period of inactivity should not exceed a certain critical duration.

Our experimental model has been important in identifying activity-dependent intracellular pathways for gene expression control. For example, how slow, but not fast, impulse patterns trigger the entry of transcription factors, such as the calcineurin-dependent NFAT factors, into the nucleus that then induce a slow phenotype. $^{50,51}$ In the brain, the decisive role of impulse patterns in synaptic plasticity, for example in driving synapses towards long-term potentiation or depression, is today taken for granted.

\section{Clinical applications}

In our 1985 paper $^{1}$ we write: "an essential neurotrophic control mechanism seems unlikely in the rat. If this conclusion can be extended to humans, then it should be possible to maintain and perhaps make some use of denervated muscles in humans by suitable electrical stimulation. If, on the other hand, neurotrophic substances are essential, such prospects 


\section{The response of denervated muscle to long-term electrical stimulation}

Eur J Trans Myol - Basic Appl Myol 2014; 24 (1): 13-19

seem less likely". Since then, much effort has been made, particularly by researchers in Vienna, to develop apparatuses and methods for percutaneous long-term stimulation of denervated leg muscles in humans with complete and irreversible lower motor neuron destruction. ${ }^{52-55}$ Remarkable improvements have been observed after 2 years of stimulation in muscle mass, fiber diameter, ultrastructure, tissue composition, and muscle force, with some parameters reaching normal values, and $25 \%$ of patients able to perform stimulation-assisted stand-up exercises. Stimulations starting after longer delays (>4 years) are much less effective. Patients use home based stimulators for daily stimulations of gluteus, thigh, and lower leg muscles on both sides. Stimulus durations are initially very long (100-300 ms), but, as recovery proceeds, the duration can be substantially reduced to allow tetanic stimulations of up to $20 \mathrm{~Hz}$ for better effects. Limitations are obvious. With no hope of reinnervation and little control of muscle contractions for most useful movements, major benefits for patients are described as cosmetic and improved comfort at sitting down due to increased muscle mass. Stimulation-induced muscle contractions and increases in muscle mass may also, like exercise, have long-term health benefits. Concerns may be raised with regard to how many patients will persevere with such demanding, long-term and, I presume, expensive, treatment, particularly in an absence of dedicated experimentalists who follow each patient closely over long periods of time. Nevertheless, the effects obtained are remarkably large, providing further evidence, now in humans, that nerve-evoked muscle impulse activity rather than a putative trophic factor is the essential regulator of (non-junctional) muscle properties.

\section{Different discoveries have different impacts}

It is interesting to compare how neuroscientists in general received the results of direct stimulation of denervated muscles with the reception of another discovery, namely long term potentiation (LTP), the phenomenon that brief activation of synapses in the brain can lead to very long-lasting increases in their efficiency of transmission, a property now generally seen as essential for the ability to learn and remember. Tim Bliss and I published the first systematic study of LTP in $1973 .{ }^{56}$ And yet it took many years for LTP to arouse the interest of most neuroscientists. As late as 1981, LTP is not mentioned in the first edition of Kandel and Schwartz influential textbook "Principles of Neuroscience". In contrast, Rosenthal and Lømo (1972) caused an immediate sensation. The issues involved were well known and most neuroscientists immediately saw their implications. The results entered textbooks and numerous review papers but then, after some years, the results and debates discussed here disappeared completely from the literature. Obviously, showing that new and unexpected phenomena exist, has greater impact than showing that something previously held to be true is wrong. The first type of discovery can lead, as in the case of LTP, to an apparently endless amount of further work, discoveries, and new insights, whereas the second type only leads to a large body of work falling by the wayside.

\section{Conclusions}

Since direct stimulation of denervated muscles with appropriate stimulation patterns can prevent the appearance of abnormal non-junctional muscle fiber properties, it seems unnecessary to postulate additional trophic factors. For every type of evidence that has been taken to support the existence of trophic factors, there are alternative and much more plausible explanations that are entirely consistent with a unique role of nerve evoked muscle impulse activity in regulating non-junctional muscle properties. Despite much effort, no one has yet identified any factor that could fulfill the roles of putative neurotrophic factors. On the contrary, when factors have been isolated from neural tissue, they induce ACh sensitivity rather than suppress it as required by the neurotrophic hypothesis. ${ }^{57}$ While reports favoring the existence of neurotrophic factors were numerous before 2000, they have now essentially disappeared from the literature, including original research papers, textbooks and handbooks, which suggest that the hypothesis is no longer arguable. Thus, the results that I presented in our paper in 1985 seem to have held up rather well.

\section{Corresponding Author}

Terje Lømo (MD, PhD), Institute of Basic Medical Sciences, University of Oslo, Sognsvannsveien 9 (Domus Medica), PO Box 1103 Blindern 0317 Oslo, Norway. E-mail: terje.lomo@basalmed.uio.no

\section{References}

1. Lømo T, Westgaard R H, Hennig R, Gundersen K. The response of denervated muscle to long-term electrical stimulation. In: Carraro U, Angelini C, eds. Proceedings of the First Abano Terme Meeting on Rehabilitation, 1985 August 28-30, Abano Terme, Padova, Italy,Cleup Padova 1985. pp 8190.

2. Tower SS. Trophic control of non-nervous tissues by the nervous system: A study of muscle and bone innervated from an isolated and quiescent region of spinal cord. J Comp Physiol 1937;67: 241-67.

3. Langley JN. Observations on denervated muscle. J Physiol 1916:50:335-44.

4. Langley JN, Kato T. The rate of loss of weight in skeletal muscle after nerve section, with some observation on the effect of stimulation and other treatment. J Physiol 1914;49:432. 


\section{The response of denervated muscle to long-term electrical stimulation}

Eur J Trans Myol - Basic Appl Myol 2014; 24 (1): 13-19

5. Miledi R. The acetylcholine sensitivity of frog muscle fibres after complete or partial denervation. J Physiol 1960;151:1-23.

6. Miledi R. Properties of regenerating neuromuscular synapses in the frog. J Physiol 1960;154:190-205.

7. Johns T R, Thesleff S. Effects of motor inactivation on the chemical sensitivity of skeletal muscle. Acta Physiol Scand 1961;51:136-41.

8. Thesleff S. Supersensitivity of skeletal muscle produced by botulinum toxin. J Physiol 1960;151; 598-607.

9. Luco JV, Eyzaguirre C. Fibrillation and hypersensitivity to $\mathrm{ACh}$ in denervated muscle: effect of length of degenerating nerve fibers. J Neurophysiol 1955; 18, 65-73.

10. Miledi R, Slater CR. On the degeneration of rat neuromuscular junctions after nerve section. J Physiol 1970;207,507-28.

11. Eccles JC. The Physiology of Synapses. Berlin Heidelberg, Germany: Springer 1964.

12. Buller AJ, Eccles JC, Eccles RM. Interactions between motoneurons and muscles in respect of the characteristic speeds of their responses. J Physiol 1960;150: 417-39.

13. Eccles JC, Eccles RM, Kozak W. Further investigations on the influence of motoneurons on the speed of muscle contraction. J Physiol 1962; 163, 324-39.

14. Lømo T, Rosenthal J. Control of ACh sensitivity by muscle activity in the rat. J Physiol 1972;221:493513.

15. Lømo T, Westgaard RH. Further studies on the control of ACh sensitivity by muscle activity in the rat. J Physiol 1975;252, 603-26.

16. Westgaard RH. Influence of activity on the passive electrical properties of denervated soleus muscle fibres in the rat. J Physiol 1975;251:683-97.

17. Lømo T, Westgaard RH, Dahl HA. Contractile properties of muscle: control by pattern of muscle activity in the rat. Proc R Soc Lond, B, Biol Sci 1974:187:99-103.

18. Westgaard RH, Lømo T. Control of contractile properties within adaptive ranges by patterns of impulse activity in the rat. J Neurosci 1988:8, 4415-26.

19. Ausoni S, Gorza L, Schiaffino S, Gundersen K, Lømo T. Expression of myosin heavy chain isoforms in stimulated fast and slow rat muscles. J Neurosci 1990;10:153-60.

20. Eken T, Gundersen K. Electrical stimulation resembling normal motor-unit activity: effects on denervated fast and slow rat muscles. J Physiol 1988;402:651-69.

21. Deshpande SS, Albuquerque EX, Guth L. Neurotrophic regulation of prejunctional and postjunctional membrane at the mammalian motor endplate. Experimental Exp Neurol 1976;53,15165.
22. Gutmann E. Neurotrophic relations. Annu Rev Physiol 1976;38:177-216. Review.

23. Jolesz F, Sréter FA. Development, innervation, and activity-pattern induced changes in skeletal muscle. Annu Rev Physiol 1981;43531-552.

24. Witzemann V, Brenner HR, Sakmann, B. Neural factors regulate $\mathrm{AChR}$ subunit mRNAs at rat neuromuscular synapses. J Cell Biol 1991;114:12541.

25. Grinnell AD. Trophic interaction between nerve and muscle. In: Engel AG, Franzini-Armstrong C, eds. Myology. New York, NY: McGraw-Hill 1994. pp 303-32.

26. Nicholls JG, Martin AR, Wallace BG, Fuchs PA. From Neuron to Brain. PediaPress, 2001.

27. Connor EA, McMahan UJ. Cell accumulation in the junctional region of denervated muscle. J Cell Biol ; 104:109-20.

28. Murray MA, Robbins N. Cell proliferation in denervated muscle: identity and origin of dividing cells. Neuroscience 1982:7,1823-33.

29. Murray MA, Robbins N. Cell proliferation in denervated muscle: time course, distribution and relation to disuse. Neuroscience 1982;7:1817-22.

30. Jones R, Vrbová G. Two factors responsible for the development of denervation hypersensitivity. J Physiol 1974;236, 517-38.

31. Arancio O, Buffelli M, Cangiano A, Pasino E. Nerve stump effects in muscle are independent of synaptic connections and are temporally correlated with nerve degeneration phenomena. Neuroscience letters 1992;146,1-4.

32. Lømo T, Westgaard RH. Control of ACh sensitivity in rat muscle fibers. Cold Spring Harbor symposia on quantitative biology 1976;40,263-74.

33. Cangiano A, Lutzemberger L. Partial denervation affects both denervated and innervated fibers in the mammalian skeletal muscle. Science 1977;196, 542-5.

34. Cangiano A, Lutzemberger L. Partial denervation in inactive muscle affects innervated and denervated fibres equally. Nature 1980;285, 233-5.

35. Pasino E, Buffelli M, Arancio, et al. Effects of long-term conduction block on membrane properties of reinnervated and normally innervated rat skeletal muscle. J Physiol 1996;497:457-72.

36. Buffelli M, Pasino E, Cangiano A. Paralysis of rat skeletal muscle equally affects contractile properties as does permanent denervation. J Muscle Res Cell Motil 1997;18:683-95.

37. Pasino E, Buffelli M, Busetto G, Cangiano A. Use of dexamethasone with TTX block of nerve conduction shows that muscle membrane properties are fully controlled by evoked activity. Brain Res 1997;770:242-7.

38. Cangiano A. Acetylcholine supersensitivity: the role of neurotrophic factors. Brain Res 1973;58:255-9. 


\section{The response of denervated muscle to long-term electrical stimulation}

Eur J Trans Myol - Basic Appl Myol 2014; 24 (1): 13-19

39. Lømo T. Neurotrophic control of colchicine effects on muscle? Nature 1974;249:473-4.

40. McMahan UJ. The agrin hypothesis. Cold Spring Harbor symposia on quantitative biology 1990;55:407-18.

41. Thoenen H, Edgar D. Neurotrophic factors. Science 1985;229:238-42.

42. Fitzsimonds RM, Poo MM. Retrograde signaling in the development and modification of synapses. Physiol Rev 1998;78:143-70.

43. Lewin GR, Barde YA. Physiology of the neurotrophins. Annu Rev Neurosci 1996;19:289317.

44. Oppenheim RW. Neurotrophic survival molecules for motoneurons: an embarrassment of riches. Neuron 1996;17:195-7.

45. Park H, Poo MM. Neurotrophin regulation of neural circuit development and function. Nat Rev Neurosci 2013;14,7-23. doi: 10.1038/nrn3379. Review.

46. Lu H, Park H, Poo M-M. Spike-timing-dependent BDNF secretion and synaptic plasticity. Philos Trans R Soc Lond B Biol Sci 2013;369(1633):20130132. doi: 10.1098/rstb. 2013.0132. Print 2014 Jan 5.

47. Pette D, Smith ME, Staudte HW, Vrbová G. Effects of long-term electrical stimulation on some contractile and metabolic characteristics of fast rabbit muscles. Pflugers Arch 1973;338:257-72.

48. Salmons S, Sréter FA. Significance of impulse activity in the transformation of skeletal muscle type. Nature 1976;263:30-4.

49. Eerbeek O, Kernell D, Verhey BA. Effects of fast and slow patterns of tonic long-term stimulation on contractile properties of fast muscle in the cat. $\mathbf{J}$ Physiol 1984:352, 73-90.
50. McCullagh KJ, Calabria E, Pallafacchina G, et al. NFAT is a nerve activity sensor in skeletal muscle and controls activity-dependent myosin switching. Proc Natl Acad Sci U S A 2004;101:10590-5. Epub 2004 Jul 9.

51. Tothova J Blaauw B, Pallafacchina $G$, et al. NFATc1 nucleo-cytoplasmic shuttling is controlled by nerve activity in skeletal muscle. J. Cell. Sci. 2006;119:1604-11. Epub 2006 Mar 28.

52. Kern H, Carraro U, Adami N, et al. Home-based functional electrical stimulation rescues permanently denervated muscles in paraplegic patients with complete lower motor neuron lesion. Neurorehabil Neural Repair. 2010;24:709-21. doi: 10.1177/1545968310366129. Epub 2010 May 11

53. Kern H, Boncompagni S, Rossini K, et al. Longterm denervation in humans causes degeneration of both contractile and excitation-contraction coupling apparatus, which is reversible by functional electrical stimulation (FES): a role for myofiber regeneration? J Neuropathol Exp Neurol. 2004;63:919-31.

54. Boncompagni S, Kern H, Rossini K et al. Structural differentiation of skeletal muscle fibers in the absence of innervation in humans. Proc Natl Acad Sci U S A. 2007;104:19339-44. Epub 2007 Nov 27.

55. Hofer C, Mayr W, Stöhr H, et al. A stimulator for functional activation of denervated muscles. Artif Organs 2002;26:276-9.

56. Bliss TV, Lømo T. Long-lasting potentiation of synaptic transmission in the dentate area of the anaesthetized rabbit following stimulation of the perforant path. J. Physiol 1973;232:331-56.

57. Younkin SG, Brett RS, Davey B, Younkin L. Substances moved by axonal transport and released by nerve stimulation have an innervation-like effect on muscle. Science 1978;200:1292-5. 